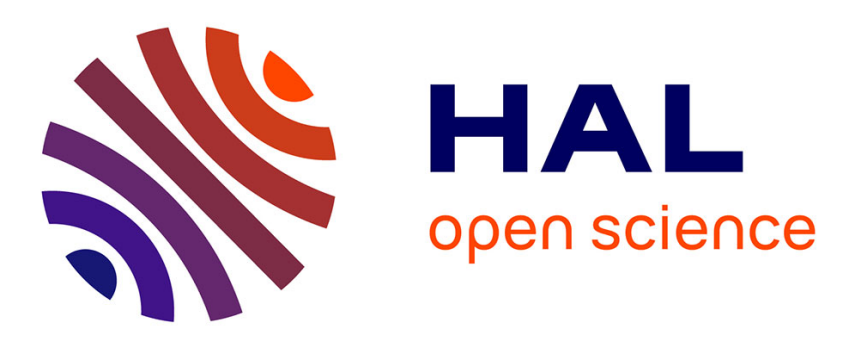

\title{
Characterization of a Static Magnetic Field with Two-Photon Rotational Spectroscopy of Cold Trapped $\mathrm{HD}+$ \\ Florin Lucian Constantin
}

\section{- To cite this version:}

Florin Lucian Constantin. Characterization of a Static Magnetic Field with Two-Photon Rotational Spectroscopy of Cold Trapped HD+. 2020 Joint Conference of the IEEE International Frequency Control Symposium and International Symposium on Applications of Ferroelectrics (IFCS-ISAF), Jul 2020, Keystone, United States. pp.1-4, 10.1109/IFCS-ISAF41089.2020.9234880 hal-03054382

\section{HAL Id: hal-03054382 \\ https://hal.science/hal-03054382}

Submitted on 11 Dec 2020

HAL is a multi-disciplinary open access archive for the deposit and dissemination of scientific research documents, whether they are published or not. The documents may come from teaching and research institutions in France or abroad, or from public or private research centers.
L'archive ouverte pluridisciplinaire HAL, est destinée au dépôt et à la diffusion de documents scientifiques de niveau recherche, publiés ou non, émanant des établissements d'enseignement et de recherche français ou étrangers, des laboratoires publics ou privés.

$$
\text { Copyright }
$$




\title{
Characterization of a Static Magnetic Field with Two-Photon Rotational Spectroscopy of Cold Trapped $\mathrm{HD}^{+}$
}

\author{
Florin Lucian Constantin \\ Laboratoire PhLAM, CNRS UMR 8523 \\ CNRS, University Lille 1 \\ Villeneuve d'Ascq, France \\ FL.Constantin@univ-lille1.fr
}

\begin{abstract}
Two-photon rotational spectroscopy of cold trapped $\mathrm{HD}^{+}$ions may be exploited for characterization of the magnitude and orientation of a static magnetic field in the ion trap. The experimental setup and the approach for calibration of the magnetic field vector are described. A sensitivity at the $10^{-11} \mathrm{~T}$ level may be reached with Zeeman spectroscopy of a hyperfine component of the $(v, L)=(0,0) \rightarrow(0,2)$ transition. The orientation of a magnetic field with a magnitude at the $1 \mu \mathrm{T}$ level may be characterized with an uncertainty better than 30 mrad.
\end{abstract}

Keywords-two-photon spectroscopy, trapped ions, hydrogen molecular ions, Zeeman shift, ab-initio calculation, vector magnetometry

\section{INTRODUCTION}

Precise sensing of the magnetic fields has applications in geophysics [1], medical imaging [2,3], and precision physics measurements [4]. The atomic magnetometers exploiting alkali atoms vapours are extremely sensitive [5], but their response, based on Larmor precession, is intrinsically scalar. This work evaluates the potential for SI-traceable full characterization of a static magnetic field from precision measurements of transitions of trapped molecular ions compared with the predictions of the state-of-the-art ab-initio energy level calculations. Precisely, the energy levels of the hydrogen molecular ions (HMI), the simplest molecular quantum systems, were predicted with quantum electrodynamics (QED) ab-initio calculations reaching a fractional accuracy at the $10^{-12}$ level [6]. The shifts of the energy levels due to various external fields were also predicted by ab-initio calculations [7,8]. Particularly, the effects of a static magnetic field on the $\mathrm{HD}^{+}$energy levels were evaluated in the nonrelativistic approximation at the order $\alpha^{2}$. The ab-initio calculations allowed identifying and exploiting for precision measurements transitions which are insensitive to the magnetic field and to its orientation with respect to the polarization of the radiation field. The rovibrational and rotational spectroscopy of trapped and sympathetically cooled $\mathrm{HD}^{+}$ions was performed by resonantenhanced multiphoton dissociation (REMPD). The Dopplerlimited infrared spectroscopy allowed a fractional uncertainty at the $10^{-9}$ level [9]. A significant increase in the accuracy and resolution can be provided by the Doppler-free spectroscopy. The rotational spectroscopy of $\mathrm{HD}^{+}$in the Lamb-Dicke regime allowed $\mathrm{kHz}-$ level resolution and a fractional accuracy at the $10^{-10}$ level [10]. A double-resonance two-photon spectroscopy scheme of $\mathrm{HD}^{+}$may provide fractional accuracy and resolution at the $10^{-12}$ level or better [11].
In precision measurements, a static magnetic field, generated with a set of coils, allows to define the quantization axis of the molecular ions and to split their energy levels. This contribution proposes to exploit the scalar response obtained from the $\mathrm{HD}^{+}$ions by two-photon Zeeman spectroscopy to characterize the magnitude and orientation of the magnetic field.

\section{THEORY AND SPECTROSCOPY OF $\mathrm{HD}^{+}$}

The rovibrational energy levels of $\mathrm{HD}^{+}$in its ground electronic state are calculated as the nonrelativistic Schrödinger energy plus a series expansion of QED correction terms [6]. At high resolution, the energy levels reveal a hyperfine structure described with the coupling of the proton $\overrightarrow{\mathrm{I}}_{\mathrm{p}}$, deuteron $\overrightarrow{\mathrm{I}}_{\mathrm{d}}$, and electron $\overrightarrow{\mathrm{S}}_{\mathrm{e}}$ spins with the rotational angular momentum $\overrightarrow{\mathrm{L}}$ to yield $\overrightarrow{\mathrm{J}}$, the total angular momentum: $\overrightarrow{\mathrm{F}}=\overrightarrow{\mathrm{S}}_{\mathrm{e}}+\overrightarrow{\mathrm{I}}_{\mathrm{p}} ; \overrightarrow{\mathrm{S}}=\overrightarrow{\mathrm{F}}+\overrightarrow{\mathrm{I}}_{\mathrm{d}} ; \overrightarrow{\mathrm{J}}=\overrightarrow{\mathrm{L}}+\overrightarrow{\mathrm{S}}$. The hyperfine eigenstates $\mid v L F S J>$, labelled with the quantum numbers for the vibration $\mathrm{v}$ and for the angular momentum coupling scheme, are calculated using a Breit-type spin Hamiltonian and accurate variational Coulomb wavefunctions [12]. A small magnetic field is used to define the quantization axis and to split the hyperfine states in magnetic subcomponents, labelled with the quantum number $J_{Z}$ of the projection of $\vec{J}$ on the quantization axis. The Zeeman shift of an energy level in an external static magnetic field may be described with a quadratic dependence in function of the magnetic field magnitude [7]. The total energy of a magnetic level can be expressed as :

$$
\begin{aligned}
E\left(v, L, F, S, J, J_{z}\right)= & E_{r v}(v, L)+E_{h f}(v, L, F, S, J) \\
& +\Delta E_{z}\left(v, L, F, S, J, J_{z} ; B\right)
\end{aligned}
$$

in function of the rovibrational energy $E_{r v}(v, L)$, the hyperfine energy $E_{h f}(v, L, F, S, J)$, and the Zeeman shift $\Delta E_{z}\left(v, L, F, S, J, J_{z} ; B\right)$, respectively.

Accurate measurements of the $\mathrm{HD}^{+}$transitions can be performed with an experimental setup based on cold trapped ions $[9,10]$. Precisely, $\sim 10^{2} \mathrm{HD}^{+}$ions and $\sim 10^{3} \mathrm{Be}^{+}$ions are trapped together in a radiofrequency trap. The $\mathrm{Be}^{+}$ions are laser cooled with a $313 \mathrm{~nm}$ laser. The electrostatic interactions between two species allow sympathetical cooling of the $\mathrm{HD}^{+}$ions. When the secular motion of the $\mathrm{HD}^{+}$ions is excited in the trap, the increase in fluorescence 
of the laser-cooled $\mathrm{Be}^{+}$ions is used to monitor the number of the trapped $\mathrm{HD}^{+}$ions. A static magnetic field, controlled with three coil pairs, driven with three independent current sources, is applied to the ion trap. The measurements proposed in this contribution are based on the double resonance spectroscopy scheme discussed in [11], that addresses the two-photon rotational transition in the vibrational ground state $(\mathrm{v}, \mathrm{L})=(0,0) \rightarrow(0,2)$ and the twophoton rovibrational transition $(\mathrm{v}, \mathrm{L})=(0,0) \rightarrow(2,0)$. The detection is performed by the photodissociation of the $(\mathrm{v}, \mathrm{L})=(2,0)$ level with a $175 \mathrm{~nm}$ laser. The Doppler-free spectroscopy is performed with two counterpropagating waves tuned around the rovibrational transition at 55.909 $\mathrm{THz}$, and the rotational transition at $1.968 \mathrm{THz}$, respectively (Fig. 1). The two-photon transition rates between the magnetic levels are derived using the two-photon operator formalism and the second-order time-dependent perturbation theory. The interaction with the blackbody radiation at room temperature recycles continuously the population among the rotational levels of the ground vibrational state. Moreover, the population in an excited energy level decays radiatively. The change of the population in the rovibrational levels is calculated with a set of rate equations in order to derive the lineshapes of the two-photon resonances [11]. The full-width half measured linewidth is $12.1 \mathrm{~Hz}$ for the rovibrational line and $0.447 \mathrm{~Hz}$ for the rotational line, by assuming a twophoton rotational transition rate of $2000 \mathrm{~s}^{-1}$, a two-photon rovibrational transition rate of $10 \mathrm{~s}^{-1}$, a dissociation rate of $200 \mathrm{~s}^{-1}$ and a REMPD time of $10 \mathrm{~s}$.

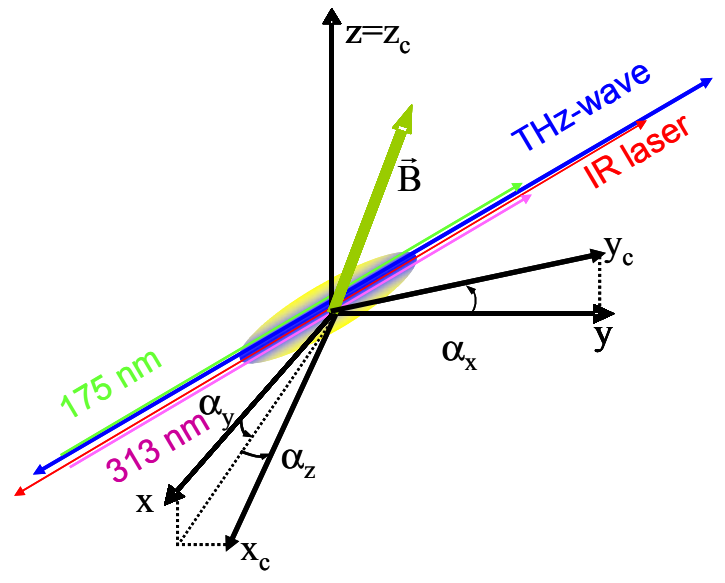

Fig. 1. The experimental setup and the reference coordinate frames.

The fractional frequency uncertainty is estimated with the Allan variance for the quantum projection noise limit :

$$
\sigma_{y}(\tau)=\frac{1}{\pi Q \sqrt{N_{i o n}}} \times \sqrt{\frac{T_{c}}{\tau}}
$$

expressed in function of the quality factor of the two-photon transition $Q=f_{2 p h} / \Delta f_{H W H M}$ in terms of the half-linewidth determined previously. The cycle time $T_{c}$ is associated to a single measurement with $N_{i o n}$ ions at the two-photon resonance. Successive measurements are averaged during an interrogation time $\tau$. Assuming the case of single ion spectroscopy experiment with $T_{c}=\tau$, the frequency uncertainty for $(\mathrm{v}, \mathrm{L})=(0,0) \rightarrow(2,0)$ line is estimated at $1.9 \mathrm{~Hz}$ and for $(\mathrm{v}, \mathrm{L})=(0,0) \rightarrow(0,2)$ line at $71 \mathrm{mHz}$.
The Zeeman shift of a transition $\mid \mathrm{v}, \mathrm{L}, \mathrm{F}, \mathrm{S}, \mathrm{J}_{\mathrm{Z}} \mathrm{J}_{\mathrm{Z}}>=\left(\mathrm{n}, \mathrm{J}_{\mathrm{z}}\right) \rightarrow\left(\mathrm{n}^{\prime}, \mathrm{J}_{\mathrm{z}}\right)^{\prime}$ may be calculated approximatively with a quadratic dependence :

$$
\begin{array}{r}
f_{n, n^{\prime}}(\mathrm{B})-f_{n, n^{\prime}}(\mathrm{B}=0)=\eta_{B}\left(t_{n}, q_{n}, r_{n}, t_{n^{\prime}}, q_{n^{\prime}}, r_{n^{\prime}}, J_{z}, J_{z}^{\prime}\right) B \\
+\eta_{B^{2}}\left(t_{n}, q_{n}, r_{n}, t_{n^{\prime}}, q_{n^{\prime}}, r_{n^{\prime}}, J_{z}, J_{z}^{\prime}\right) B^{2}
\end{array}
$$

in function of the magnitude of the magnetic field $B$ and a set of six theoretical parameters $\left\{U_{t h}\right\}=\left\{t_{n}, q_{n}, r_{n}, t_{n^{\prime}}, q_{n^{\prime}}, r_{n^{\prime}}\right\}$ that were calculated ab-initio for the relevant hyperfine energy levels of $\mathrm{HD}^{+}$in [7]. The favoured (intense) twophoton electric dipole (E1-E1) transitions obey the selection rules $\Delta \mathrm{F}=\Delta \mathrm{S}=0, \Delta \mathrm{J}=0, \pm 2$. The linear and quadratic Zeeman shift coefficients are calculated and displayed in Table I for a selection of magnetic subcomponents of the rotational and rovibrational two-photon transitions. The subcomponents of the rotational transition, in general more sensitive than the subcomponents of the rovibrational transition, are suitable for magnetic field characterization. When the $\mathrm{THz}$ source used for two-photon spectroscopy is referenced to a frequency standard, this approach allows absolute calibration of the magnetic field in the ion trap, using Zeeman spectroscopy measurements, ab-initio calculations of the $\mathrm{HD}^{+}$energy levels and of their Zeeman shifts.

TABLE I. ZEEMAN SHIFT COEFFICIENTS FOR SELECTED COMPONENTS OF THE TWO-PHOTON ROTATIONAL AND ROVIBRATIONAL TRANSITIONS.

\begin{tabular}{|c|c|c|c|}
\hline $\mathbf{v}, \mathbf{L}, \mathbf{F}, \mathbf{S}, \mathbf{J} ; \mathbf{J}_{\mathbf{z}}$ & $\mathbf{v}^{\prime}, \mathbf{L}^{\prime}, \mathbf{F}^{\prime}, \mathbf{S}^{\prime}, \mathbf{J}^{\prime} ; \mathbf{J}_{\mathbf{z}}$ & $\Delta \boldsymbol{\eta}_{\mathbf{B}}(\mathbf{k H z} / \mathbf{T})$ & $\Delta \boldsymbol{\eta}_{\mathbf{B} \mathbf{2}}\left(\mathbf{M H z} / \mathbf{T}^{\mathbf{2}}\right)$ \\
\hline $0,0,1,0,0 ; 0$ & $2,0,1,0,0 ; 0$ & 0 & 40900 \\
\hline $0,0,0,1,1 ; \pm 1$ & $2,0,0,1,1 ; \pm 1$ & $\mp 155$ & 4700 \\
\hline $0,0,1,2,2 ; \mp 2$ & $0,2,1,2,4 ; 0$ & \pm 6992320 & 1855850 \\
\hline $0,0,1,1,1 ; \mp 1$ & $0,2,1,1,3 ; \pm 1$ & \pm 6123100 & 397050 \\
\hline
\end{tabular}

\section{CHARACTERIZATION OF A STATIC MAGNETIC FIELD}

This part will describe a procedure for measuring the magnitude and orientation of a static magnetic field in the Cartesian Laboratory Coordinate Frame $\operatorname{LCF}\left(\vec{e}_{x}, \vec{e}_{y}, \vec{e}_{z}\right)$, by using two-photon Zeeman spectroscopy of $\mathrm{HD}^{+}$(Fig. 1). The three coil pairs define a Coil Coordinate Frame $C C F\left(\vec{e}_{c, x}, \vec{e}_{c, y}, \vec{e}_{c, z}\right)$ which is not necessary orthogonal. The relative orientations of the $C C F$ axis relative to $L C F$ are defined with the Euler angles : $\left(\alpha_{z}, \pi / 2+\alpha_{y}\right)$ for $\vec{e}_{c, x}$, $\left(\pi / 2, \pi / 2-\alpha_{x}\right)$ for $\vec{e}_{c, y}$, and $(0,0)$ for $\vec{e}_{c, z}$, respectively. A slight nonorthogonality of $C C F$ is accounted with the small angles $\alpha_{x}, \alpha_{y}, \alpha_{z}<<1$.

The measurement of the Zeeman shift $\delta f$ of a magnetic subcomponent of the two photon rotational transition $\left|v L F S J J_{Z}>=\right| n, J_{Z}>\rightarrow \mid n^{\prime}, J^{\prime}{ }_{z}>$ allows to calculate the magnitude of the magnetic field in function of the relevant values for the theoretical parameters $\left\{U_{t h}\right\}$, by inverting eq. (3). A choice between the two values of the magnetic field derived from the second-order equation may be made by comparison with the value obtained with an accurate magnetometer. The uncertainty of the magnetic field may be calculated with the error propagation formula, in function of the contribution from the frequency shift measurement uncertainty 
$\sigma_{\text {exp,rot }}=0.1 \mathrm{~Hz}$, estimated with the quantum projection noise limit, and the contributions from the theoretical parameters, which are assumed correlated and having the same uncertainty $\sigma_{t h, q, r}=50 \mathrm{MHz} / \mathrm{T}^{2}, \sigma_{t h, t}=5 \mathrm{kHz} / \mathrm{T}$. Here, the Zeeman spectroscopy of the subcomponent $\left|\mathrm{v}, \mathrm{L}, \mathrm{F}, \mathrm{S}, \mathrm{J}, \mathrm{J}_{\mathrm{Z}}>=\right| 0,0,1,2,2,-2>\rightarrow \mid 0,2,1,2,4,0>$, having the highest Zeeman shift of $717.791 \mathrm{kHz}$ at $B=10^{-4} \mathrm{~T}$, is exploited for measuring the magnetic field. The dependence of the uncertainty with the magnitude of the magnetic field in the ion trap is plotted in Figure 2. The sensitivity of the twophoton Zeeman spectroscopy method is given by the experimental uncertainty of $1.4 \times 10^{-11} \mathrm{~T}$. Moreover, a magnetic field smaller than $10^{-4} \mathrm{~T}$ may be calibrated with a precision better than $7 \times 10^{-11} \mathrm{~T}$.

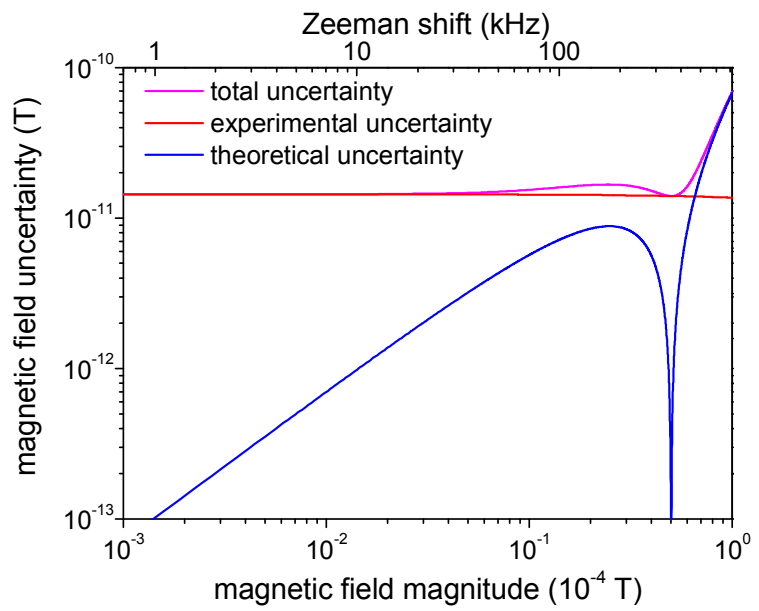

Fig. 2. Total uncertainty (magenta line), the contribution from the frequency measurement (red line), and the contribution from the theoretical calculation (blue line) of the magnitude of the magnetic field measured by Zeeman spectroscopy of the $\left|\mathrm{v}, \mathrm{L}, \mathrm{F}, \mathrm{S}, \mathrm{J}, \mathrm{J}_{\mathrm{Z}}>=\right| 0,0,1,2,2,-2>\rightarrow \mid 0,2,1,2,4,0>$ transition of $\mathrm{HD}^{+}$. The frequency uncertainty is estimated with the quantum projection noise-limited instability for a single-ion experiment with integration time $1 \mathrm{~s}$ and cycle time $1 \mathrm{~s}$.

The directional measurements of the magnetic field are determined by a set of experimental parameters: three currents $\left(I_{1}, I_{2}, I_{3}\right)$ that drive independently the coil pairs in Helmholtz configuration, the current-to-field parameters of the coil pairs $\left(k_{1}, k_{2}, k_{3}\right)$, three angles that define the relative orientation of the coil pairs $\left(\alpha_{x}, \alpha_{y}, \alpha_{z}\right)$, and the offset magnetic field components $\left(B_{01}, B_{02}, B_{03}\right)$, due for example to the Earth's magnetic field. As discussed previously, the magnitude of the magnetic field can be expressed in function of the experimental frequency of a two-photon transition $f_{\text {exp }, i}$ shifted by the Zeeman effect from the theoretical value $f_{t h, i}$ calculated at zero-field in proportion with the linear Zeeman shift coefficient $\eta_{i}$ :

$$
\left\|\vec{B}\left(\left\{V_{k}\right\}_{i} ; I_{1, i}, I_{2, i}, I_{3, i}\right)\right\|=\frac{f_{\text {exp }, i}-f_{t h, i}}{\eta_{i}}
$$

This equation assumes linear approximation. The set of nine parameters $\left\{V_{k}\right\}_{i}=\left\{k_{1}, k_{2}, k_{3}, \alpha_{x}, \alpha_{y}, \alpha_{z}, B_{01}, B_{02}, B_{03}\right\}$ has to be determined from Zeeman spectroscopy measurements. The theoretical value of the zero-field frequency is expressed as the sum $f_{t h, i}=f_{r o t, i}+f_{h f, i}$ of the rotational frequency and the hyperfine frequency. A nonlinear least-squares adjustment of $\mathrm{N}$ Zeeman subcomponents can be exploited for absolute calibration of the experimental parameters $\left\{V_{k}\right\}$ (at least $\mathrm{N}=9$ measurements are required). In order to estimate the uncertainties, the magnetic field dependence of eq. (4) is linearized around the design values of the parameters $\left\{V_{k ; 0}\right\}$ :

$$
\begin{aligned}
\left\|\vec{B}\left(\left\{V_{k}\right\}_{i} ; I_{1, i}, I_{2, i}, I_{3, i}\right)\right\|=\left\|\vec{B}\left(\left\{V_{k ; 0}\right\}_{i} ; I_{1, i}, I_{2, i}, I_{3, i}\right)\right\| \\
+\left.\sum_{k} \frac{\partial\left\|\vec{B}\left(\left\{V_{k}\right\}_{i} ; I_{1, i}, I_{2, i}, I_{3, i}\right)\right\|}{\partial V_{k}}\right|_{\left\{V_{k}\right\}=\left\{V_{k ; 0}\right\}}\left(V_{k}-V_{k ; 0}\right)
\end{aligned}
$$

The $9 \times 9$ covariance matrix $G$ of the adjusted parameters may be estimated in function of the $\mathrm{N} \times \mathrm{N}$ covariance matrix $X$ of the input data $b_{i}=\left(f_{\text {exp }, i}-f_{t h, i}\right) / \eta_{i}$, and the $\mathrm{N} \times 9$ Jacobian matrix $A=\left\{\left.\left(\partial\left\|\vec{B}_{i}\right\| / \partial V_{k}\right)_{i, k}\right|_{\left\{V_{k}\right\}=\left\{V_{k ; 0}\right\}}\right\}$ :

$$
G=\left(A^{T} X^{-1} A\right)^{-1}
$$

The uncertainties of the input data are estimated as the quadratic sum of the contributions from the experimental uncertainty of the Zeeman subcomponent $\delta f_{\text {exp }, i}=71 \mathrm{mHz}$, and from the theoretical uncertainties of the rotational frequency $\delta f_{r o t, i}=10^{-12} \times f_{r o t, i}$, the hyperfine frequency $\delta f_{h f, i}=0.5 \mathrm{kHz}$, and the Zeeman shift coefficient $\delta \eta_{i}=5 \mathrm{kHz} / \mathrm{T}$ :

$$
\delta b_{i}=\sqrt{\left(\frac{\delta f_{\text {exp }, i}}{\eta_{i}}\right)^{2}+\left(\frac{\delta f_{r o t, i}}{\eta_{i}}\right)^{2}+\left(\frac{\delta f_{h f, i}}{\eta_{i}}\right)^{2}+\left(\frac{\delta \eta_{i}\left(f_{r o t, i}+f_{h f, i}\right)}{\eta_{i}^{2}}\right)^{2}}
$$

The nondiagonal elements of the $X=\left\{x_{i j}\right\}$ matrix arise from the covariances between the theoretical parameters, expressed as :

$$
\begin{aligned}
x_{i j} & =\left(\frac{\delta f_{\text {exp }, i}}{\eta_{i}}\right)^{2} \times \delta_{\mathrm{ij}}+\frac{\delta f_{r o t, i} \delta f_{r o t, j}}{\eta_{i} \eta_{j}}+\frac{\delta f_{h f, i} \delta f_{h f, j}}{\eta_{i} \eta_{j}} \\
& +\left(\frac{\delta \eta_{i}\left(f_{r o t, i}+f_{h f, i}\right)}{\eta_{i}^{2}}\right)\left(\frac{\delta \eta_{j}\left(f_{r o t, j}+f_{h f, j}\right)}{\eta_{j}^{2}}\right)
\end{aligned}
$$

Let's consider an experiment designed with the following values of the parameters: $k_{1}=k_{2}=k_{3}=-10^{-4} \mathrm{~T} / \mathrm{A}$, $\alpha_{x}=\alpha_{y}=\alpha_{z}=0.05 \mathrm{rad}$. and $B_{01}=3.82 \times 10^{-7} \mathrm{~T}$, $B_{02}=2.09 \times 10^{-5} \mathrm{~T}, B_{03}=4.34 \times 10^{-5} \mathrm{~T}$, respectively. The calibration is performed by Zeeman spectroscopy of the $\left|\mathrm{v}, \mathrm{L}, \mathrm{F}, \mathrm{S}, \mathrm{J}, \mathrm{J}_{\mathrm{Z}}\right\rangle=|0,0,1,2,2,-2>\rightarrow| 0,2,1,2,4,0>\mathrm{HD}^{+}$transition. The Zeeman two-photon frequencies are measured for 27 sets $\left(I_{1}, I_{2}, I_{3}\right)=\left(I_{o f f}+n_{1} I_{0}, I_{\text {off } 2}+n_{2} I_{0}, I_{\text {off } 3}+n_{3} I_{0}\right)$ of current intensities, where $I_{0}=1 \mathrm{~A}, I_{\text {off } 1}=3.82 \mathrm{~mA}, I_{\text {off } 2}=208$ 
$\mathrm{mA}, I_{\text {off } 3}=422 \mathrm{~mA}$, and $n_{1,2,3}=0,1,2$. The uncertainties of the values of the experimental parameters, that can be determined by the nonlinear adjustment, are estimated with eq. (4-8) :

$$
\begin{aligned}
& \delta k_{1}=5.4 \times 10^{-12} \mathrm{~T} / \mathrm{A}, \delta k_{2}=1.6 \times 10^{-11} \mathrm{~T} / \mathrm{A}, \\
& \delta k_{3}=3.0 \times 10^{-11} \mathrm{~T} / \mathrm{A}, \quad \delta \alpha_{x}=1.6 \times 10^{-7} \mathrm{rad}, \\
& \delta \alpha_{y}=1.1 \times 10^{-7} \mathrm{rad}, \delta \alpha_{z}=7.9 \times 10^{-8} \mathrm{rad}, \\
& \delta B_{01}=1.3 \times 10^{-11} \mathrm{~T}, \delta B_{02}=1.2 \times 10^{-11} \mathrm{~T}, \\
& \delta B_{03}=1.5 \times 10^{-11} \mathrm{~T},
\end{aligned}
$$

Cancellation of the Earth's magnetic field in the ion trap is realized using the values of the current intensities that minimize eq. (4) $: I_{\text {null } 1}=-6.34 \mathrm{~mA}, I_{\text {null } 2}=230 \mathrm{~mA}, I_{\text {null } 3}=$ $434 \mathrm{~mA}$. The uncertainty in setting the magnitude of the magnetic field is calculated with the root sum of squares of the contributions from the uncertainties of setting the current intensities (assumed at a fractional value of $10^{-3}$ of each current intensity), and from the uncertainties of the experimental parameters determined previously by Zeeman spectroscopy. Particularly, the uncertainty in setting the null magnetic field in the ion trap is estimated at $3.9 \times 10^{-8} \mathrm{~T}$, if the theoretical uncertainties are neglected. In addition, a conservative estimation of the orientation uncertainty for a magnetic field with a given magnitude may be derived by assuming that the total magnetic field uncertainty is perpendicular to the magnetic field direction. If the three pairs of coils are driven by $(-6.33 \mathrm{~mA}, 240 \mathrm{~mA}, 444 \mathrm{~mA})$ currents, the magnitude of the magnetic field is $1.7 \times 10^{-6} \mathrm{~T}$ and the orientation uncertainty is $30 \mathrm{mrad}$.

\section{CONCLUSION}

This contribution describes the experimental setup and the calibration technique for the magnitude and the orientation of the magnetic field in an ion trap using precision Zeeman spectroscopy of $\mathrm{HD}^{+}$ions. The Zeeman shift for a selected hyperfine component of a two-photon rotational transition is exploited for the determination of the magnitude of a static magnetic field with an uncertainty at the $10^{-11} \mathrm{~T}$ level. The approach allows coil calibration that enables to predict the magnitude and orientation of the magnetic field in function of the coil currents. The approach allows cancellation of the Earth's magnetic field at the $39 \mathrm{nT}$ level. The orientation uncertainty of a magnetic field of 1.7 $\mu \mathrm{T}$ is better than $30 \mathrm{mrad}$.

\section{REFERENCES}

[1] W.F. Stuart, "Earth's field magnetometry," Rep. Prog. Phys., vol. 35, pp. 803-881, May 1972.

[2] E. Boto et al, "On the potential of a new generation of magnetometers for MEG: A beamformer simulation study," PLoS One., vol. 11, p. e0157655, August 2016.

[3] O. Alem, et al, "Fetal magnetocardiography measurements with an array of microfabricated optically pumped magnetometers," Phys. Med. Biol., vol. 60, p. 4797, June 2015.

[4] D. Budker and D.F.J. Kimball, Optical Magnetometry, Oxford: Cambridge University Press, 2013

[5] I.K. Kominis et al, "A subfemtotesla multichannel atomic magnetometer," Nature, vol. 422, pp. 596-599, April 2003.

[6] V.I. Korobov, L. Hilico, and J.-P. Karr, "Fundamental transitions and ionization energies of the hydrogen molecular ions with few ppt uncertainty," Phys. Rev. Lett., vol. 118, p. 233001, June 2017.

[7] D. Bakalov, V.I. Korobov, and S. Schiller, "Magnetic field effects in the transitions of the $\mathrm{HD}^{+}$molecular ion and precision spectroscopy," J. Phys. B., vol. 44, p. 025003, January 2011.

[8] S. Schiller, D. Bakalov, A.K. Bekbaev, and V.I. Korobov, "Static and dynamic polarizability and the Stark and blackbody-radiation frequency shifts of the molecular hydrogen ions $\mathrm{H}_{2}{ }^{+}, \mathrm{HD}^{+}$, and $\mathrm{D}_{2}{ }^{+}$," Phys. Rev. A, vol. 89, p. 052521, May 2014.

[9] J. Biesheuvel, J.-P. Karr, L. Hilico, K.S.E. Eikema, W. Ubachs, and J.C.J. Koelemeij, "Probing QED and fundamental constants through laser spectroscopy of vibrational transitions in $\mathrm{HD}^{+}$," Nat. Commun., vol. 7, p. 10385, January 2016.

[10] S. Alighanbari, M.G. Hansen, V.I. Korobov, and S. Schiller, "Rotational spectroscopy of cold and trapped molecular ions in the Lamb-Dicke regime," Nat. Phys., vol. 14, pp. 555-559, March 2018.

[11] F.L. Constantin, "Double-resonance two-photon spectroscopy of hydrogen molecular ions for improved determination of fundamental constants," IEEE Trans. Instrum. Meas., vol. 68, pp. 2151-2159, March 2019.

[12] D. Bakalov, V.I. Korobov, and S. Schiller, "High-precision calculation of the hyperfine structure of the $\mathrm{HD}^{+}$ion," Phys. Rev. Lett., vol. 97, p. 243001, December 2006. 